\title{
Development of a validated patient-reported symptom metric for pediatric Eosinophilic Esophagitis: qualitative methods
}

\author{
James P Franciosi ${ }^{1 *}$, Kevin A Hommel ${ }^{2}$, Charles W DeBrosse ${ }^{3}$, Allison B Greenberg ${ }^{4}$, Alexandria J Greenler ${ }^{3}$, \\ J Pablo Abonia ${ }^{3}$, Marc E Rothenberg ${ }^{3}$ and James W Varni ${ }^{5}$
}

\begin{abstract}
Background: Previous attempts to measure symptoms in pediatric Eosinophilic Esophagitis (EoE) have not fully included patients and parents in the item development process. We sought to identify and validate key patient self-reported and parent proxy-reported outcomes (PROs) specific to EoE.

Methods: We developed methodology for focus and cognitive interviews based on the Food and Drug Administration (FDA) guidelines for PROs, the validated generic PedsQL ${ }^{\mathrm{TM}}$ guidelines, and the consolidated criteria for reporting qualitative research (COREQ). Both child (ages 8-12 and 13-18) and parent-proxy (ages 2-4, 5-7, 8-12, and 13-18) interviews were conducted.

Results: We conducted 75 interviews to construct the new instrument. Items were identified and developed from individual focus interviews, followed by cognitive interviews for face and content validation. Initial domains of symptom frequency and severity were developed, and open-ended questions were used to generate specific items during the focus interviews. Once developed, the instrument construct, instructions, timeframe, scoring, and specific items were systematically reviewed with a separate group of patients and their parents during the cognitive interviews.

Conclusions: To capture the full impact of pediatric EoE, both histologic findings and PROs need to be included as equally important outcome measures. We have developed the face and content validated Pediatric Eosinophilic Esophagitis Symptom Score (PEESSTM V2.0). The PEESSTM V2.0 metric is now undergoing multisite national field testing as the next iterative instrument development phase.
\end{abstract}

\section{Background}

In pediatric eosinophilic esophagitis (EoE) there can be a wide range of symptoms: from the easily recognizable presentations of food bolus impaction and dysphagia to the less obvious feeding disorders and abdominal pain [1]. Most previous efforts to develop a questionnaire to measure pediatric EoE symptoms have focused on correlations between non-validated symptom scores and histologic outcomes, specifically peak esophageal eosinophil counts $[2,3]$. To date, there have been few validated instruments that capture symptoms as patient and parent proxyreported outcomes (PROs) developed through patient

\footnotetext{
* Correspondence: james.franciosi@cchmc.org

'Division of Gastroenterology, Hepatology and Nutrition, Cincinnati

Children's Hospital Medical Center, Cincinnati, OH, USA

Full list of author information is available at the end of the article
}

focus interviews and cognitive interviews as recommended by the FDA and specific to pediatric EoE $[1,4]$.

Pentiuk et al. developed the non-validated Pediatric Eosinophilic Esophagitis Symptom Score (PEESS version 1.0) utilizing expert opinion and focusing on correlations between symptom scores and histologic outcomes. In their study, Pentiuk and colleagues demonstrated that subjects with untreated EoE had higher PEESS scores than treated subjects; however, symptom score and histology were only weakly correlated [5]. Aceves and colleagues have also recently developed a symptom score through the modification of a metric utilized for acid peptic disorders [3]. However, this non-validated tool was again developed through expert opinion alone and focused on correlations to histology. In Aceves's study, the total symptom score was higher among patients with EoE and gastroesophageal

\section{Biomed Central}


reflux disease (GERD) than control patients $(\mathrm{P}<0.001)$. However, only symptoms of dysphagia and anorexia/early satiety were capable of significantly discriminating EoE from GERD ( $\mathrm{P}<0.01)$. Patients with chief complaints other than dysphagia and anorexia did not have histology findings that directly correlated with the results of the modified symptoms scale.

These prior efforts to develop a symptom score for EoE depending solely on expert opinion have not fully taken into account patient perceptions of symptom severity or response to treatment, which are increasingly important to improving health outcomes and quality of life [4]. Therefore, there is a significant need for severity indices to be developed as patient self-report and parent proxy-reported outcomes. Well designed and validated PROs have been increasingly recognized as key outcome measures for the treatment of chronic disease over the past decade. For example, the recent pediatric asthma randomized clinical trial for the medication ciclesonide utilized both patient symptoms and quality of life (QOL) metrics as key outcome measures and demonstrated improvements in QOL and symptoms scores in the treated groups relative to the placebo controls [6]. The National Institutes of Health identified PROs as key components in the clinical research "toolbox" and have launched the Patient Reported Outcomes Measurement Information System (PROMIS, http://www.nihpromis.org/default.aspx) in an attempt to provide healthcare workers and researchers with instruments to objectively measure the disease characteristics that patients and their families deem critically important for their day-to-day health [7-14]. It is clear that a pediatric EoE symptom score would provide a valuable new tool with which to better analyze the outcomes in pediatric EoE that are relevant to families.

As a first phase in the development process of a new PRO instrument, content validity must be established. The FDA guidelines on PRO development state that, "item generation should include input from the target patient population to establish the items that reflect the concept of interest and contribute to its evaluation." To capture outcomes from the patients' perspective, in 2009 the FDA recommended that it is important to establish content validity through patient focus groups/interviews and cognitive think aloud and cognitive debriefing protocols before evaluating other measurement properties. Basic PRO validation requires feedback from patients and their parents early in the instrument development process to determine if the instrument's item content captures the disease features that pediatric patients and parents believe are important for the instrument to measure. Content validity is supported using qualitative research methodologies to investigate whether the PRO instrument and its respective items measure the symptoms of interest from the patients' perspective. These qualitative methods are an essential part of the instrument development process from the beginning of the process, and subsequently testing other measurement properties will not replace or rectify problems with eliciting patient and parent-proxy focused outcomes from the initiation phase [7]. In 2008, Flood and colleagues described their use of cognitive interviews for a new pediatric EoE metric (Symptom Questionnaire for Eosinophilic Esophagitis) developed by experts in the EoE field for patients age 8-17 years of age and caregivers of EoE patients ages 2-7 years of age [15]. Given that the metric developed by Flood and colleagues was developed by expert opinion initially with patient input in a single cohort only, it may be limited in reflecting the patient experience.

As the primary objective of this study, we sought to identify key EoE patient self-reported and parent proxyreported symptoms through focus interviews, to develop an EoE PRO symptom metric, and to review the patient derived metric though cognitive interviews in a separate cohort. Content validation of the Pediatric Eosinophilic Esophagitis Symptoms Severity (PEESSTM v2.0) PRO metric was supported by this iterative process.

\section{Methods}

All study protocols were reviewed and approved by the Institutional Review board at Cincinnati Children's Hospital Medical Center (CCHMC). All research described was compliant with the CCHMC ethical guidelines for clinical research and the Helsinki Declaration (http:// www.wma.net/e/policy/b3.htm). Informed consent was obtained from all parents and assent for children ages 8-18 years of age.

FDA guidelines on PRO development, the validated generic PedsQL ${ }^{\mathrm{TM}}$ guidelines, and the consolidated criteria for reporting qualitative research (COREQ) were used to develop the methodology for content validation $[7,13,16]$.

\section{Research Team}

The research team was composed of experts in the field of EoE including specialty physicians from the fields of Allergy and Gastroenterology with advanced training in clinical research methodologies and two Ph.D. psychologists with specific expertise in qualitative research and PRO methodology. The entire team developed interview guidelines, and specific interviewers were trained in qualitative methodology. The research team reviewed transcriptions of the interview audiotapes.

\section{Participants}

Participants were identified from local and referral populations with a clinicopathologic diagnosis of EoE based on chart review and family interview. All participants had 
a clinical diagnosis of EoE, and their histology reports were reviewed to identify at least one endoscopy with $>=$ $15 \mathrm{eos} / \mathrm{hpf}$ isolated to the esophagus. As it was critical to assess patients' and families' concerns that were specific to EoE, not those related to other co-morbidities, pediatric patient participants were restricted to those with a diagnosis of EoE and without other co-morbidities, including clinical diagnoses of: gastroesophageal reflux (GERD), inflammatory bowel disease, celiac disease, psychiatric disorder, and/or therapy with psychiatric/ behavioral medication.

\section{Focus Interviews}

The methodologies for focus interviews (also termed concept elicitation interviews) have been described previously $[12,17]$. Briefly, our research team developed a script of semi-structured open-ended questions (Table 1). The content of the PEESS v1.0 was used only as a general outline for open-ended patient and parent-proxy questions. Interviewers facilitated the participants' answers by focusing discussion on the topics under consideration while being nondirective and nonevaluative. Interviews lasted approximately one hour, with age stratification in alignment with the PedsQL ${ }^{\mathrm{TM}}$ guidelines (http://www.pedsql.org), considered a gold standard for pediatric PRO metrics [12].

All sessions were audio-recorded, transcribed verbatim, and analyzed by the research team. Responses were grouped according to domains of interest, age ranges, and patient vs. parent proxy groups. Content and themes were then derived by consensus among the research team. Disagreements were minor and easily resolved by further discussion and then consensus was reached within the group.

\section{Expert Opinion}

Items derived from the focus interviews were integrated into a preliminary draft of the PEESS ${ }^{\mathrm{TM}}$ version 2.0. Local (authors) and national (listed in acknowledgements) EoE experts in the fields of allergy, gastroenterology, and psychology then reviewed this draft. To retain the focus of the PEESS ${ }^{\mathrm{TM}}$ v2.0 on patient self-reported and parent proxy-reported outcomes, experts were allowed only to discuss general concerns and suggest (not delete) additional items. This additional information was then reviewed with a separate cohort of subjects in the cognitive interview phase. This step is a critical distinction from previous EoE metrics [15].

\section{Cognitive Interviews}

Cognitive interviews were designed to elicit information regarding the clarity and rationale of the directions, individual items, domains, and response choices, as well as overall comments on the relevance and complexity of the questionnaire. Participants completed and reviewed the PEESS $^{\text {TM }}$ version 2.0 preliminary draft derived from the focus interviews and provided feedback utilizing the previously described respondent debriefing methodology [13]. Briefly, cognitive probes and items generated by focus interview subjects and national experts were assimilated into a protocol from the existing methodological literature by Varni and colleagues (Table 2). An item-by-item summary of each section of the questionnaire, including recommendations for modifications, was prepared from transcribed audiotapes and interviewer notes. Using the same methodology as described for the focus interviews, items and content generated were revised. Reading level was assessed using the Flesch Reading Easiness and the Flesch-Kincaid Grade Level scores and used to further revise question language and grammar. A summary of the content validation methodology is provided in Figure 1.

\section{Results}

Basic participant demographics of the focus interview and cognitive interview cohorts are provided in Table 3.

\section{Focus Interviews Patient Self-Report}

A total of 36 participants were interviewed, divided among children ages 8-12 and 13-18 and parents of

Table 1 Focus interview open-ended questions

\begin{tabular}{ll}
\hline Participant & Questions \\
\hline & What symptoms do you (does your child) have that you relate to EoE? \\
& Pain in chest? \\
& Burning in chest? \\
Child (Parent) & Trouble swallowing (eating food)? \\
& Womiting/throwing up? \\
& What is the most frequent symptom? How often does this occur? \\
& How often to do you call your (your child's) doctor? \\
& Because of your (your child's) symptoms, do you (s/he) have trouble in school? Work? Playing with friends? \\
& What trouble do you (your child) have eating food? \\
\hline
\end{tabular}


Table 2 Cognitive interview respondent debriefing

\begin{tabular}{ll}
\hline Subject & Question \\
\hline Directions & How would you make the directions more clear/easy to understand? \\
& When you see "the past month", what days did you include? \\
In your own words, what do you think this question is asking? & What does this question mean to you? What did you think of when answering this question? \\
& Was this question easy to understand? Are there any specific words that are difficult to understand? \\
& How would you change the words to make it more clear? \\
& Was this item hard to answer? If yes, why? \\
Domains & How did you choose your answer? \\
& In your own words, what do you think this group of questions is asking about? \\
Response & Are there any questions that do not belong in this group? \\
Choices & What do you think about the response choices? \\
Overall & How would you make the response choices clearer or easier to understand? \\
Assessment & Are there things that we forgot to ask about that you think are important? \\
& Overall thoughts/opinions of the questionnaire? \\
\hline
\end{tabular}

children ages 2-4, 5-7, 8-12, and 13-18. Children in the 8-12 and 13-18 year age groups described and discussed their symptoms related to EoE. Three focus interviews in the 5-7 year age group were also attempted. However, children in this younger age group were not able to fully describe and discuss their symptoms. Therefore, only parent proxy PRO measures were developed for children in the 2-4 and 5-7 year old age groups. Children ages 812 and 13-18 were interviewed separately from their parents. Children in the 8-12 and 13-18 year groups focused on pain and difficulties with eating food. Regarding frequency, most children described this as how "often" a particular symptom was occurring. Regarding severity, all children described this as how "bad" a particular symptom was. We were surprised to find how clearly children could discern the feeling of nausea from vomiting or abdominal pain. Most children also accurately described heartburn and regurgitation. In addition, children frequently described dysphagia as trouble swallowing or the sensation of food getting stuck.

\section{Focus Interviews Parent Proxy-Report}

Although similar to patient self-reported symptoms, parent proxy-reported concerns often differed regarding perceptions of symptom severity. In addition, parents focused more on amounts of food eaten than children did. Parents also described issues with frequency and severity of symptoms as separate from the overall question of how much of a problem a particular symptom was for their child.

\section{Expert Opinion}

National EoE experts were particularly helpful regarding dysphagia and the various ways that patients and parents describe this important symptom. In addition to items asking about trouble swallowing and feelings of food getting stuck, items were added regarding needing to drink liquids to help swallow food and extended time needed to eat food compared to others. Expert opinions were added to the PEESS ${ }^{\mathrm{TM}}$ v2.0 draft to be reviewed by the cognitive interview cohort. No items were deleted based on expert opinion.

Focus Interviews Item and Instrument Draft Development Thirty-six focus interview transcripts were analyzed. Content and themes derived by consensus among the research team are summarized in Table 4. Once developed, the instrument construct, instructions, timeframe, scoring, and items were systematically reviewed with a different group of patients and their parents during the cognitive interviews.

\section{Cognitive Interview Patient Self-Report}

A different cohort of 39 participants divided evenly among children ages 8-12 and 13-18 and parents of children ages $2-4,5-7,8-12$, and 13-18 were interviewed. Results from the separate cohort of children with EoE in the 8-12 and 13-18 year groups who participated in the cognitive interview respondent debriefing are summarized in Table 2. In general, participants thought that the PEESS $^{\text {TM }}$ version 2.0 preliminary draft was much easier to understand than the PEESS v1.0. In response to 


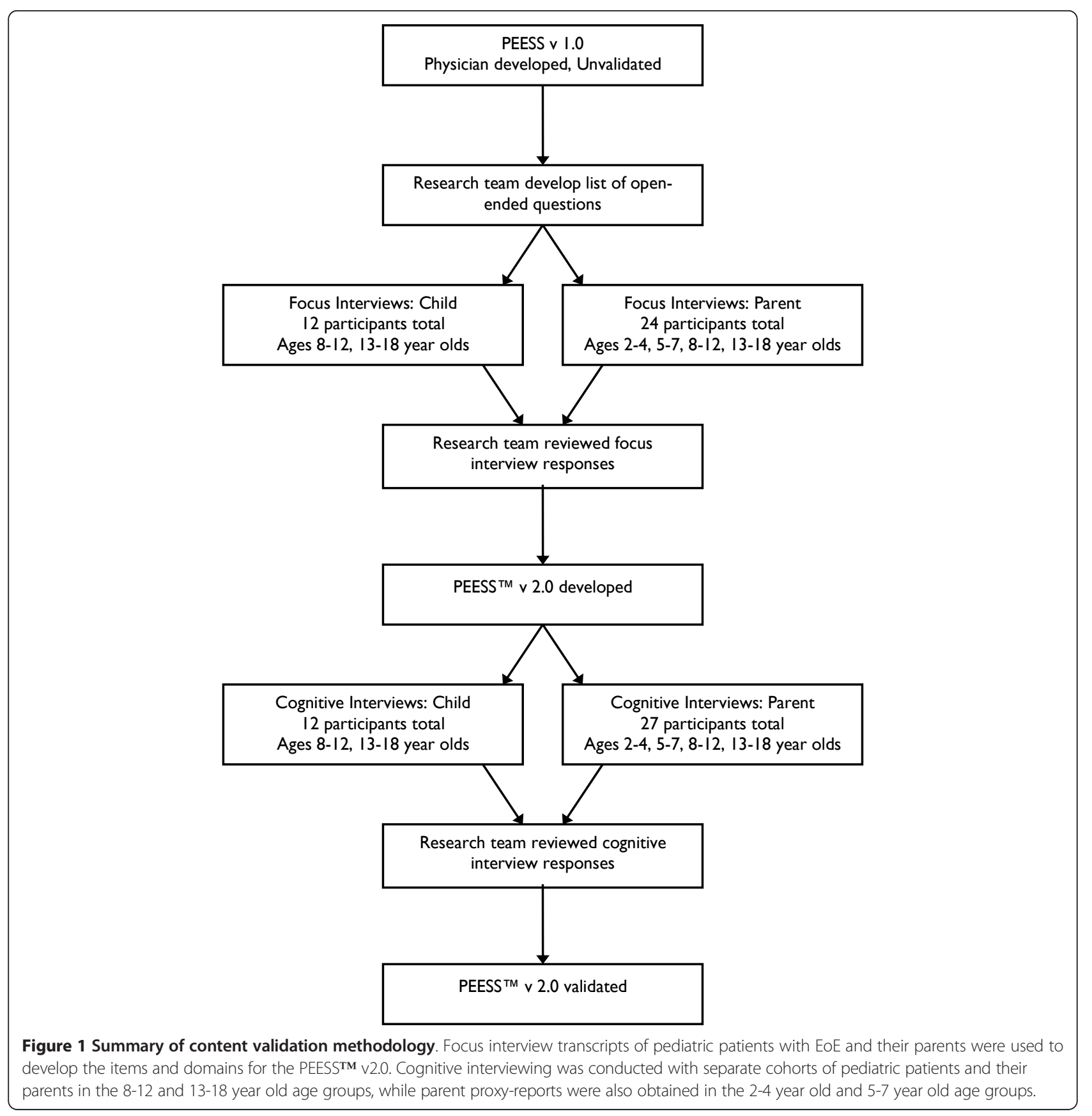

Table 3 Demographics of focus interview (FI) and cognitive interview (CI) cohorts

\begin{tabular}{lllll}
\hline & Ages 2-4 & Ages 5-7 & Ages 8-12 & Ages 13-18 \\
\hline Total sample (Fl,Cl) & $13(6,7)$ & $12(7,5)$ & $12(6,6)$ & $13(7,6)$ \\
Male \% (Fl, Cl) & $61.5 \%(3,5)$ & $83 \%(6,4)$ & $50 \%(2,4)$ & $61.5 \%(4,4)$ \\
Caucasian \% (FI, Cl) & $77 \%(5,5)$ & $83 \%(5,5)$ & $83 \%(6,4)$ & $92 \%(7,5)$ \\
Non-Hispanic \% (FI, Cl) & $92 \%(5,7)$ & $100 \%(7,5)$ & $100 \%(6,6)$ & $92 \%(6,6)$ \\
Dietary food restrictions for EoE \% (FI, Cl) & $46 \%(2,4)$ & $75 \%(5,4)$ & $50 \%(1,5)$ & $54 \%(1,6)$ \\
Swallowed steroids for EoE \% (FI, Cl) & $46 \%(4,2)$ & $42 \%(4,1)$ & $58 \%(2,5)$ & $38 \%(4,1)$ \\
Food allergies \% (Fl, Cl) & $69 \%(5,4)$ & $75 \%(5,4)$ & $58 \%(1,6)$ & $31 \%(0,4)$ \\
Atopic disease \% (Fl, Cl) & $61.5 \%(3,5)$ & $75 \%(5,4)$ & $42 \%(2,3)$ & $92 \%(6,6)$ \\
\hline
\end{tabular}


Table 4 Revised items and reasons for revision

\begin{tabular}{ll}
\hline Item & $\begin{array}{l}\text { Focus interviews } \\
\text { patient reported symptoms }\end{array}$ \\
\hline General & $\begin{array}{l}\text { Patients described how often symptoms } \\
\text { occurred and how bad symptoms as } \\
\text { different concepts } \\
\text { All } 36 \text { subjects did not understand } \\
\text { medical terminology in PEESS v1.0 }\end{array}$ \\
Chest Pain & 8 reported chest pain.
\end{tabular}

\section{PEESSTM v2.0}

Cognitive interviews:

PEESSTM v2.0 content validated

Heartburn 4 reported heartburn

1 reported reflux

4 reported "burning in chest"

Burning in chest, mouth,

or throat (heartburn)

\begin{tabular}{|c|c|}
\hline $\begin{array}{l}\text { Abdominal } \\
\text { Pain }\end{array}$ & $\begin{array}{l}5 \text { reported abdominal pain, } \\
8 \text { reported "stomachaches/pain" }\end{array}$ \\
\hline $\begin{array}{l}\text { Dysphagia/ } \\
\text { Food } \\
\text { Impaction }\end{array}$ & $\begin{array}{l}3 \text { reported dysphagia, } \\
16 \text { described "trouble swallowing" } \\
4 \text { reported feeling of "food getting stuck }\end{array}$ \\
\hline
\end{tabular}

Stomachaches or bellyaches

Trouble swallowing Needing a drink to help

swallow food

All 39 subjects agreed that the visual analog scale for symptom severity Patients preferred items in forms improved comprehension

of questions

Detailed instruction

All 39 subjects preferred PEESSTM

v2.0 over PEESS v1.0

Chest pain, aches or hurt All 39 participants understood the question and preferred to include the How often do you have chest additional descriptive words.

pain, ache, or hurt?

How bad is the chest pain, ache, or hurt?

How often do you have heartburn (burning in your chest, mouth, or

throat)?

How bad is your heartburn

(burning in your chest, mouth, or throat)?

All participants understood stomachaches and bellyaches. Even though How often do you have stomach

it is grammatically incorrect, patients preferred the separation of aches or belly aches? stomach and belly to aches.

How bad are the stomach aches or belly aches?

All participants understood trouble swallowing. How bad is the trouble Needing more time to eat than other children the same age.

Feeling like food gets stuck in throat or chest

All participants understood needing a drink to help swallow food. Some swallowing?

participants did not know that this was a symptom of EoE. How bad is the trouble All participants understood needing more time to eat than other swallowing?

children the same age. Some patients did not see this as a symptom of How often do you need to drink EoE but as a behavioral issue.

The participants agreed that how bad did not apply to this question

and there for only frequency was used

lot to help swallow your food?

All participants understood feeling like food gets stuck in throat or chest How often do you need more

time to eat than others?

Item deleted

How often do you feel like food gets stuck in your throat or chest?

Vomiting 9 reported vomiting,

5 reported "throwing up"

Throwing up (vomiting)

All participants understood throwing up

Nausea

2 reported nausea,

3 reported "feeling like they are

going to throw up but don't'

Feeling like throwing up

but didn't (nausea)

38 out of 39 participants understood the term nausea. All participants did understand the descriptive phrase for nausea.

Regurgitation 6 described as "food coming up throat".

Food coming back up throat when eating

How often do you vomit (throw

up)?

How bad is the vomiting

(throwing up)?

How often do you feel nauseous (feel like you're going to throw up, but don't)?

How bad is the nausea (feeling like you're going to throw up, but don't)?

How often does food come back up your throat when eating? How bad is the food coming back up your throat when eating? 
Table 4 Revised items and reasons for revision (Continued)

Poor 13 reported poor appetite or "not

appetite wanting to eat"

Inadequate

weight gain

Early Satiety

1 reported inadequate weight gain

reported early satiety
Not eating as much as

ther children the same

My child has/l have two or
more of these problems

this often:

Other problems. They are:
Most patients understood not eating as much as other children as a measure for poor appetite and inadequate weight gain.

The participants agreed that how bad did not apply to this question and therefore, only frequency was used

The 30 day time frame of PEESS $\vee 2.0$ does not allow for appropriate measure of inadequate weight gain.

Removed because only one patient reported early satiety as a symptom. Item Deleted All 39 subjects found this question confusing and unnecessary. It was Item Deleted removed in the final version.

All 39 subjects found this question unnecessary as all symptoms pertaining to EoE were covered. It was removed in the final version. 
questions raised about the language of the severity score response choices, a combined pictorial scale and Likert scale was presented to participants. The feedback regarding this addition was uniformly positive, with participants asserting that the draft was much easier to interpret. For items such as vomiting, nausea, and heartburn, participants thought that having explanations of these terms in parentheses allowed for easier comprehension.

\section{Cognitive Interview Parent Proxy-Report}

A separate cohort of parents of children ages 2-4, 5-7, 812 , and 13-18 was identified and interviewed independently from their children. Overall, responses to the PEESS TM version 2.0 preliminary draft were very positive, with participants reporting the response choices as much easier to understand than those in the PEESS v1.0. The addition of several questions regarding dysphagia was also positively received, and participants thought it was important to include all additional questions. The items "eating less food than others" and "needing more time to eat" were particularly important to parents of children in the 2-4 and 5-7 year age groups. Questions were raised about the overall layout of the instrument; many parents (as well as patients) thought that having frequency and severity of particular items side by side, rather than on two separate pages, would facilitate measuring each symptom comprehensively (Figure 2).

Final Data Analysis and Construction of the PEESSTM v2.0 After the 39 cognitive interviews were concluded, each interview transcript was reviewed in conjunction with the 36 focus interview transcripts ( 75 total interviews). Participants' demographics are provided in Table 3. Results are illustrated in Figure 2 and summarized in Table 4. Saturation of themes and changes were deemed achieved when no new themes emerged during the focus interviews or suggestions for changes occurred during the cognitive interviews. If two or more participants did not like a particular item or shared a particular concern, the item was reviewed and modified by the research team. We modified additional language and item constructs based on Flesch Reading Easiness and Flesch-Kincaid Grade Level [17]. For example, items 5 and 6 were changed from the grammatically correct "stomachaches" and "bellyaches" to the grammatically incorrect "stomach aches" and "belly aches." This adjustment increased the Flesch Reading Ease score and decreased the Flesch-Kincaid Grade level, suggesting that the items were easier to comprehend. Disagreements were resolved by discussion, and the PEESSTM v2.0 was developed. The metrics are shown in Additional Files 1 and 2, and are available at http://www. mapi-trust.org.

\section{Discussion}

Pediatric patient self-reported and parent proxy-reported outcomes are critical components of evaluating the impact of current and planned treatments for pediatric EoE. We report a patient self-report and parent-proxy Pediatric EoE Symptom Score (PEESS TM v2.0) metric with content-validation. The key distinction between the PEESS v1.0 compared to the PEESS ${ }^{\mathrm{TM}} \mathrm{v} 2.0$ is that the former are physician developed metrics, while the latter was developed from the words and descriptions of patients and families following FDA guidelines published in 2009.

The variety of symptoms reported by pediatric EoE patients and their families in the development of the PEESS $^{\mathrm{TM}}$ v2.0 was surprising. The existing literature reports that adult EoE patients often describe varying degrees of dysphagia, whereas children often describe pain without dysphagia as their only symptom $[1,3]$. Characterization of dysphagia from multiple perspectives was critical to capturing this important symptom. Patients and parent proxies were often aware that they (or their child) were experiencing dysphagia as assessed by items such as "trouble swallowing" or "food getting stuck while eating." However, the addition of items including "taking a long time to eat food" and "needing to drink a lot of water while eating food" captured the more subtle descriptions of dysphagia. Utilizing two distinct cohorts for the focus interviews and cognitive interviews also yielded invaluable information and allowed further refinement of patient self-reported and parent proxy-reported perspectives, achieving content saturation. In particular, clarification and increased reading ease of directions, addition of a combined pictorial scale and Likert scale, language modification, and changes in instrument layout were all developed from the cognitive interviews.

One particular concern regarding parent-proxy reported outcomes is that they may not be acceptable to the current FDA guidelines regarding product labeling specifications. For FDA-endorsed clinical trials that would use the PEESS ${ }^{\mathrm{TM}}$ v2.0, it may be that presently we are only able to obtain specific PRO based labeling for pediatric self-report ages 8-18 years of age. Current opinion at the FDA is that parent observer tools for children 2-7 years of age are recommended to include only symptoms clearly observable by the parent, such as: vomiting, increased time needed to eat, and drinking a large amount of liquids to swallow food. Therefore, only a subscale of questions may able to be used for clinical trials in children 2-7 years of age. However, it is also vital to recognize that FDA opinion for labeling purposes regarding PROs has changed significantly in the past several decades. It is highly possible that the current thinking on 


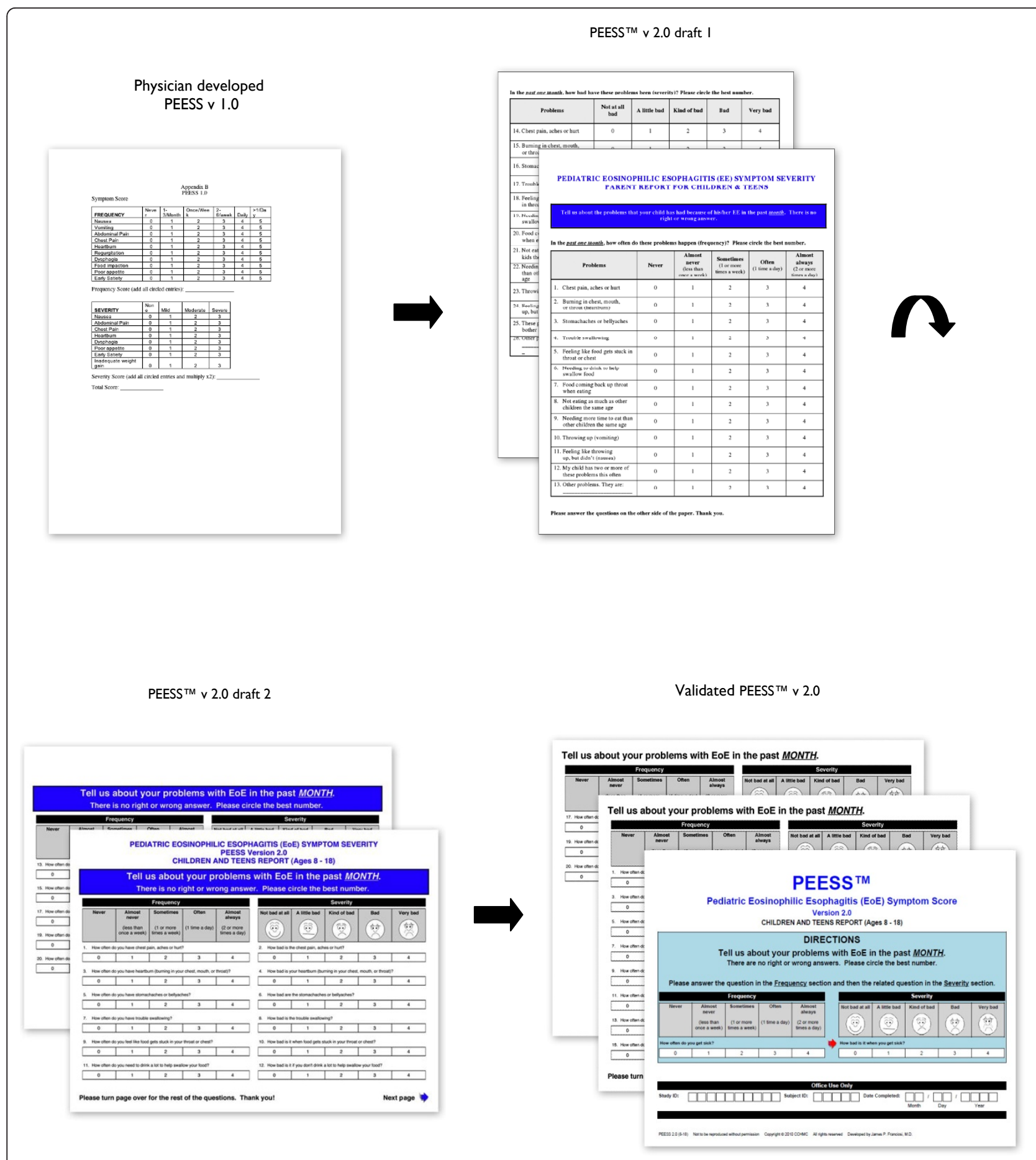

Figure 2 From the PEESS v1.0 to the validated PEESSTM $\mathbf{v}$ 2.0. The instrument layout and overall design went through several iterative drafts based on patient and parent-proxy feedback through focus interviews and separate cognitive interviews. For example, participants favored presenting frequency and severity of particular items side by side rather than on two separate pages.

parent-proxy metrics (widely supported by many psychometricians and clinicians alike) may also change. In future studies, we will be conducting responsiveness testing of the PEESS ${ }^{\mathrm{TM}}$ v2.0 metric, further assessing the parent proxy-report metric and specific subscales. Finally, the PEESS ${ }^{\mathrm{TM}}$ v2.0 is intended for use both in and outside of the clinical trial setting. We felt that all of the symptoms important to patients and their parents as proxy are 
important to capture in order to reflect the true clinical picture of symptomatology.

In developing a metric for pediatric EoE, it is important to ensure that all patient areas of concern are fully elicited - a concept often termed item saturation, and a key reason why we performed our 75 patient and parent proxy interviews in two stages and two separate cohorts. Item saturation was achieved for each question and questionnaire as a whole during the cognitive interviews for each age range. Specific questions are provided in Table 2, including: "how would you change the questions words to make it easier to understand" and the overall assessment question "are there things that we forgot to ask about that you feel are important?" These questions were asked to the patients in the 8-18 year old age ranges and to the parents in the 2-18 year old age ranges. In response, the landscape questionnaire format and front page directions were changed early on, and the final draft was reviewed by patients in this format. This is an important distinction from the Flood etal. metric and the PEESS v1.0 in that items were generated by the patients in one cohort and then reviewed by a second cohort. However, the item content if the PEESSTM v2.0 is supported by the symptom overlap reported by Flood et al. and other investigators [3,15,18].

Another controversial area in PRO development is recall period. Some may argue that a one month recall period for the PEESS ${ }^{\mathrm{TM}}$ v2.0 is too long to accurately assess change. The use of a one month recall period has been well described by Varni and colleagues [19-26]. In addition, the Mayo Dysphagia Questionnaire developed by Romero and colleagues was shown not to be responsive over a 14 day recall period, but responsive over a 30 day (1 month) recall period [27]. In future studies we will be conducting responsiveness testing of the PEESSTM v2.0 metric and will be assessing different recall periods 1 week, 2 week and 1 month. One alternative to improve patient accuracy in symptom reporting by a shorter recall period and also allow for a total responsive period of 30 days would be to collect symptom scores on a weekly basis for 4 weeks, and then report a 30 day summation score. Some have also advocated for the use of daily assessments through electronic diaries. We considered using daily electronic diaries, but we have found the patient response-burden too high, and that these diaries are impractical for any but the most highly funded and closely regulated clinical trials. The PEESS ${ }^{\mathrm{TM}} \mathrm{v} 2.0$ is intended for use both in and outside the clinical trial setting as a simple, yet effective patient-derived metric that does not cause undue burden on patients, families, clinicians, or researchers.

There are several important limitations to this study. Although the PEESS ${ }^{\mathrm{TM}}$ v2.0 metric represents an important step forward for the field of pediatric EoE, further testing and modifications will be needed. Despite item saturation, there may be additional EoE symptoms that have not been included. In addition, generalizability to non-Caucasians and to patients with co-morbidities may be a potential concern. For the PEESS ${ }^{\mathrm{TM}}$ v2.0 metric development and cognitive interviews, we felt it critical to assess patients' and families' concerns specific to EoE, not those related to other co-morbidities. In the next phase of the PEESS ${ }^{\mathrm{TM}}$ v2.0 metric development, we will test the metric construct and reliability in over 200 patients and 250 parents across a variety of demographics and co-morbidities. The fourth phase of psychometric testing will test the responsiveness of the PEESS $^{\text {TM }}$ v2.0 metric, key to assessing the potential performance of the PEESS ${ }^{\mathrm{TM}}$ v2.0 in clinical trials. The PEESS $^{\text {TM }}$ v2.0 metrics are available at http://www.mapitrust.org.

\section{Conclusions}

Currently, pediatric EoE is assessed using number of eosinophils per high powered field in an esophageal endoscopic biopsy specimen as the primary outcome variable. Symptoms are at best reported as secondary outcomes utilizing physician directed questions, without any attention to PROs $[2,28]$. It is currently welldescribed that the severity of histologic inflammation in EoE as measured by tissue eosinophil counts may not directly relate to the degree of symptom severity [5]. For example, initial data in the pediatric Reslizumab (Cinquil $^{\mathbb{R}}$ ) study suggests that patient symptoms and health related quality of life (HRQOL) do not correlate with histologic esophageal inflammation [29]. In a condition without known risk of malignancy or reduced life expectancy, PROs need to be equally if not more important outcome measures compared to histologic disease activity. Face and content validation of the PEESSTM v2.0 are important first steps to establishing patient self-report and parent proxy-report symptom assessments as key factors in pediatric EoE for patients, families, researchers, and care providers alike.

\section{Additional material}

Additional File 1: Supplement 1 - Pediatric Eosinophilic Esophagitis (EoE) Symptom Score (PEESS ${ }^{\mathrm{TM}}{ }^{\mathrm{TM}}$ v2.0) Children and Teens Report (Ages 8-18)

Additional File 2: Supplement 2 - Pediatric Eosinophilic Esophagitis (EoE) Symptom Score (PEESSTMTM v2.0) Parent Report for Children and Teens (Ages 2-18).

\section{Acknowledgements}

We thank Drs. Glenn Furuta, Mary Klinnert, Seema Aceves, and Jonathan Spergel for their thoughtful review of these instruments. We thank Dr. Carol Henderson for her editorial assistance. This work has been generously supported by the CDHNF, TAP and AstraZeneca Eosinophilic Esophagitis 
Young Investigators Award, the Campaign Urging Research for Eosinophilic Disorders (CURED), the Food Allergy Project, PHS Grant P30 DK078392, and the Buckeye Foundation.

\section{Author details}

'Division of Gastroenterology, Hepatology and Nutrition, Cincinnati Children's Hospital Medical Center, Cincinnati, OH, USA. ${ }^{2}$ Center for the Promotion of Treatment Adherence and Self-Management, Division of Behavioral Medicine and Clinical Psychology, Cincinnati Children's Hospital Medical Center, Cincinnati, OH, USA. ${ }^{3}$ Division of Allergy and Immunology, Cincinnati Children's Hospital Medical Center, Cincinnati, OH, USA. ${ }^{4}$ Clinical Trials Office, Cincinnati Children's Hospital Medical Center, Cincinnati, $\mathrm{OH}$, USA. ${ }^{5}$ Department of Pediatrics, College of Medicine, Department of Landscape Architecture and Urban Planning, College of Architecture, Texas A\&M University, College Station, TX, USA.

\section{Authors' contributions}

JPF substantially contributed to study conception and design, as well as acquisition, analysis, and interpretation of data, drafting and revision of the manuscript, and acquisition of funding. KAH substantially contributed to study conception and design, analysis and interpretation of data, and revision of the manuscript. CWD substantially contributed to acquisition, analysis, and interpretation of data, as well as revision of the manuscript. ABG substantially contributed to study conception and design, acquisition, analysis, and interpretation of data, as well as drafting and revision of the manuscript. AJG substantially contributed to study conception and design, acquisition, analysis, and interpretation of data, as well as drafting and revision of the manuscript. JPA substantially contributed to study conception and design, revision of the manuscript, and acquisition of funding. MER contributed to analysis and interpretation of data, revision of the manuscript, and acquisition of funding. JWV substantially contributed to study conception and design and revision of the manuscript. All authors read and approved the final manuscript.

\section{Competing interests}

Cincinnati Children's Hospital Medical Center and Dr. James P. Franciosi hold the copyright and the trademark for the PEESS ${ }^{\mathrm{TM}} \mathrm{V} 2.0$ and may receive financial compensation from the Mapi Research Trust, a nonprofit research institute that charges distribution fees to for-profit companies that use the PEESS ${ }^{T M} \vee 2.0^{\text {TM }}$

Received: 7 April 2011 Accepted: 18 November 2011

Published: 18 November 2011

\section{References}

1. Furuta GT, Liacouras CA, Collins MH, Gupta SK, Justinich C, Putnam PE, Bonis P, Hassall E, Straumann A, Rothenberg ME: Eosinophilic esophagitis in children and adults: a systematic review and consensus recommendations for diagnosis and treatment. Gastroenterology 2007, 133(4):1342-1363.

2. Konikoff MR, Noel RJ, Blanchard C, Kirby C, Jameson SC, Buckmeier BK Akers R, Cohen MB, Collins MH, Assa'ad AH, et al: A randomized, doubleblind, placebo-controlled trial of fluticasone propionate for pediatric eosinophilic esophagitis. Gastroenterology 2006, 131(5):1381-1391.

3. Aceves SS, Newbury RO, Dohil MA, Bastian JF, Dohil R: A symptom scoring tool for identifying pediatric patients with eosinophilic esophagitis and correlating symptoms with inflammation. Ann Allergy Asthma Immunol 2009, 103(5):401-406.

4. Klinnert MD: Psychological impact of eosinophilic esophagitis on children and families. Immunol Allergy Clin North Am 2009, 29(1):99-107, X.

5. Rothenberg ME, Pentiuk S, Putnam PE, Collins MH: Dissociation Between Symptoms and Histological Severity in Pediatric Eosinophilic Esophagitis. $J$ Pediatr Gastr Nutr 2009, 48(2):152-160.

6. Pedersen S, Potter P, Dachev S, Bosheva M, Kaczmarek J, Springer E, Dunkel J, Engelstatter R: Efficacy and safety of three ciclesonide doses vs placebo in children with asthma: the RAINBOW study. Respir Med 2010, 104(11):1618-1628.

7. Cella D, Yount S, Rothrock N, Gershon R, Cook K, Reeve B, Ader D, Fries JF, Bruce B, Rose M: The Patient-Reported Outcomes Measurement
Information System (PROMIS): progress of an NIH Roadmap cooperative group during its first two years. Med Care 2007, 45(5 Suppl 1):S3-S11.

8. DeWalt DA, Rothrock N, Yount S, Stone AA: Evaluation of item candidates: the PROMIS qualitative item review. Med Care 2007, 45(5 Suppl 1):S12-21.

9. Aletaha D: From the item to the outcome: the promising prospects of PROMIS. Arthritis Res Ther 2010, 12(1):104.

10. Einav S, Hersch M: The "PrOMIS" of things to come. Crit Care Med 2007, 35(4):1193-1194

11. Fries JF, Krishnan E, Bruce B: Items, Instruments, Crosswalks, and PROMIS. J Rheumatol 2009, 36(6):1093-1095.

12. Walsh TR, Irwin DE, Meier A, Varni JW, DeWalt DA: The use of focus groups in the development of the PROMIS pediatrics item bank. Quality of Life Research 2008, 17(5):725-735.

13. Irwin DE, Varni JW, Yeatts K, DeWalt DA: Cognitive interviewing methodology in the development of a pediatric item bank: a patient reported outcomes measurement information system (PROMIS) study. Health and Quality of Life Outcomes 2009, 7.

14. Reeve BB, Hays RD, Bjorner JB, Cook KF, Crane PK, Teresi JA, Thissen D, Revicki DA, Weiss DJ, Hambleton RK, et al: Psychometric evaluation and calibration of health-related quality of life item banks: plans for the Patient-Reported Outcomes Measurement Information System (PROMIS). Med Care 2007, 45(5 Suppl 1):S22-31.

15. Flood EM, Beusterien KM, Amonkar MM, Jurgensen CH, Dewit OE, Kahl LP, Matza LS: Patient and caregiver perspective on pediatric eosinophilic esophagitis and newly developed symptom questionnaires*. Curr Med Res Opin 2008, 24(12):3369-3381.

16. Ronen GM, Rosenbaum P, Law M, Streiner DL: Health-related quality of life in childhood disorders: a modified focus group technique to involve children. Quality of Life Research 2001, 10(1):71-79.

17. Tong A, Sainsbury P, Craig J: Consolidated criteria for reporting qualitative research (COREQ): a 32-item checklist for interviews and focus groups. Int J Qual Health Care 2007, 19(6):349-357.

18. Pentiuk S, Putnam PE, Collins MH, Rothenberg ME: Dissociation between symptoms and histological severity in pediatric eosinophilic esophagitis. J Pediatr Gastroenterol Nutr 2009, 48(2):152-160.

19. Schurman JV, Wu YP, Grayson P, Friesen CA: A pilot study to assess the efficacy of biofeedback-assisted relaxation training as an adjunct treatment for pediatric functional dyspepsia associated with duodenal eosinophilia. J Pediatr Psychol 2010, 35(8):837-847.

20. Southwell BR, Clarke MCC, Chase JW, Gibb S, Hutson JM: Improvement of quality of life in children with slow transit constipation after treatment with transcutaneous electrical stimulation. J Pediatr Surg 2009, 44(6):1268-1273.

21. Nuboer R, Borsboom GJJM, Zoethout JA, Koot HM, Bruining J: Effects of insulin pump vs. injection treatment on quality of life and impact of disease in children with type 1 diabetes mellitus in a randomized, prospective comparison. Pediatr Diabetes 2008, 9(4):291-296.

22. Schwimmer JB, Middleton MS, Deutsch R, Lavine JE: A phase 2 clinical trial of metformin as a treatment for non-diabetic paediatric non-alcoholic steatohepatitis. Aliment Pharmacol Ther 2005, 21(7):871-879.

23. Zeller MH, Reiter-Purtill J, Ratcliff MB, Inge TH, Noll JG: Two-year trends in psychosocial functioning after adolescent Roux-en-Y gastric bypass. Surg Obes Relat Dis 2011.

24. Parekh AD, Thomas JC, Trusler L, Ankerst DP, Deshpande JK, Adams MC, Pope JCt, Brock JW: Prospective evaluation of health related quality of life for pediatric patients with ureteropelvic junction obstruction. J Urol 2008, 180(5):2171-2175, discussion 2175-2176.

25. Thornburg CD, Calatroni A, Panepinto JA: Differences in health-related quality of life in children with sickle cell disease receiving hydroxyurea. $J$ Pediatr Hematol Oncol 2011, 33(4):251-254.

26. Fullerton $G$, Tyler $C$, Johnston CA, Vincent JP, Harris GE, Foreyt JP: Quality of life in Mexican-American children following a weight management program. Obesity (Silver Spring) 2007, 15(11):2553-2556.

27. McElhiney J, Lohse MR, Arora AS, Peloquin JM, Geno DM, Kuntz MM, Enders FB, Fredericksen M, Abdalla AA, Khan Y, et al: The Mayo Dysphagia Questionnaire-30: documentation of reliability and validity of a tool for interventional trials in adults with esophageal disease. Dysphagia 2010, 25(3):221-230. 
28. Dohil R, Newbury R, Fox L, Bastian J, Aceves S: Oral viscous budesonide is effective in children with eosinophilic esophagitis in a randomized, placebo-controlled trial. Gastroenterology 2010, 139(2):418-429.

29. Walsh GM: Reslizumab for pediatric eosinophilic esophagitis. Immunotherapy-Uk 2010, 2(4):461-465.

Pre-publication history

The pre-publication history for this paper can be accessed here: http://www.biomedcentral.com/1471-230X/11/126/prepub

doi:10.1186/1471-230X-11-126

Cite this article as: Franciosi et al:: Development of a validated patientreported symptom metric for pediatric Eosinophilic Esophagitis: qualitative methods. BMC Gastroenterology 2011 11:126.

\section{Submit your next manuscript to BioMed Central} and take full advantage of:

- Convenient online submission

- Thorough peer review

- No space constraints or color figure charges

- Immediate publication on acceptance

- Inclusion in PubMed, CAS, Scopus and Google Scholar

- Research which is freely available for redistribution

Submit your manuscript at www.biomedcentral.com/submit
() BioMed Central 